\title{
Visible Imaging Fourier Transform Spectrometer: Design and Calibration
}

E.H. Wishnow, R. Wurtz, S. Blais-Ouellett, K.H. Cook, D. Carr, I. Lewish, F. Grandmont, C.W. Stubbs

This article was submitted to SPIE Astronomical Telescopes and Instrumentation, Waikoloa, HI, August 22-28, 2002

\section{September 19, 2002}

U.S. Department of Energy

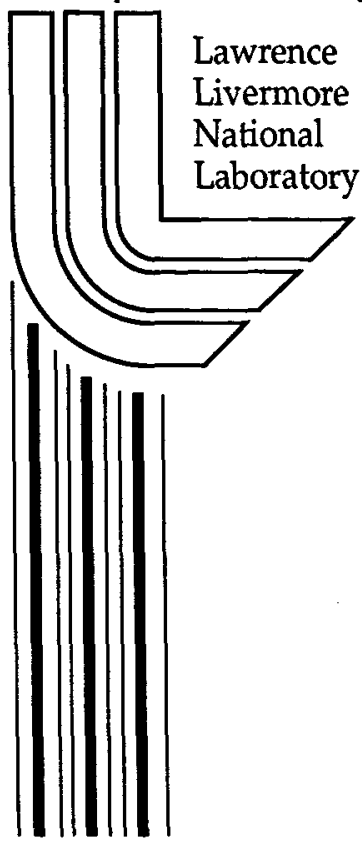




\section{DISCLAIMER}

This document was prepared as an account of work sponsored by an agency of the United States Government. Neither the United States Government nor the University of California nor any of their employees, makes any warranty, express or implied, or assumes any legal liability or responsibility for the accuracy, completeness, or usefulness of any information, apparatus, product, or process disclosed, or represents that its use would not infringe privately owned rights. Reference herein to any specific commercial product, process, or service by trade name, trademark, manufacturer, or otherwise, does not necessarily constitute or imply its endorsement, recommendation, or favoring by the United States Government or the University of California. The views and opinions of authors expressed herein do not necessarily state or reflect those of the United States Government or the University of California, and shall not be used for advertising or product endorsement purposes.

This is a preprint of a paper intended for publication in a journal or proceedings. Since changes may be made before publication, this preprint is made available with the understanding that it will not be cited or reproduced without the permission of the author.

This work was performed under the auspices of the United States Department of Energy by the University of California, Lawrence Livermore National Laboratory under contract No. W-7405-Eng-48.

This report has been reproduced directly from the best available copy.

Available electronically at http://www.doc.gov/bridge

Available for a processing fee to U.S. Department of Energy

And its contractors in paper from

U.S. Department of Energy

Office of Scientific and Technical Information

P.O. Box 62

Oak Ridge, TN 37831-0062

Telephone: (865) 576-8401

Facsimile: (865) 576-5728

E-mail: reports@adonis.osti.gov

Available for the sale to the public from

U.S. Department of Commerce

National Technical Information Service

5285 Port Royal Road

Springfield, VA 22161

Telephone: (800) 553-6847

Facsimile: (703) 605-6900

E-mail: orders@ntis.fedworld.gov

Online ordering: http://www.ntis.gov/ordering.htm

\section{OR}

Lawrence Livermore National Laboratory

Technical Information Department's Digital Library

http://www.llnl.gov/tid/Library.html 


\title{
Visible Imaging Fourier Transform Spectrometer: Design and Calibration
}

\author{
E.H. Wishnow, ${ }^{a}$ R. Wurtz, ${ }^{a}$ S. Blais-Ouellette, ${ }^{b}$ K.H. Cook, ${ }^{a}$ D. Carr,,${ }^{a}$ I. Lewis, ${ }^{a}$ F. Grandmont, $,{ }^{c}, d$ \\ and C.W. Stubbs ${ }^{e}$ \\ ${ }^{a}$ Physics and Advanced Technology Directorate, \\ Lawrence Livermore National Laboratory \\ ${ }^{b}$ Departement de Physique, Universite de Montreal \\ ${ }^{c}$ Departement de Physique, Universite Laval \\ ${ }^{d}$ ABB-Bomem Inc. \\ ${ }^{e}$ Department of Astronomy, University of Washington
}

\begin{abstract}
We present details of the design, operation and calibration of an astronomical visible-band imaging Fourier transform spectrometer (IFTS). This type of instrument produces a spectrum for every pixel in the field of view where the spectral resolution is flexible. The instrument is a dual-input/dual-output Michelson interferometer coupled to the 3.5 meter telescope at the Apache Point Observatory. Imaging performance and interferograms and spectra from calibration sources and standard stars are discussed.
\end{abstract}

Keywords: imaging Fourier Transform spectrometer, imaging spectrometer, multi-object spectrometer

\section{INTRODUCTION}

We have built a visible band imaging Fourier transform spectrometer (IFTS) and are conducting astronomical observations with this new instrument (see Wurtz et al. this conference ${ }^{1}$ ). This type of instrument is a very powerful new tool for astronomy as spectra are obtained for every pixel in the field of view, and the spectral resolution is flexible. An IFTS is an ideal instrument for programs which require spectroscopic information across the entire field. It differs from multi-object grating spectrometers in that the entire CCD is used for imaging and consequently large fields can be observed. Furthermore, since no slits are involved, the IFTS has an overall throughput which is comparable to that of a camera.

This project began with a design study for an instrument for the Next Generation Space Telescope ${ }^{2}$ and the current instrument was built as a demonstration that an imaging Fourier transform spectrometer could precisely maintain mirror positions over integration times that extend over many minutes. Because the backgrounds for a space-borne infrared telescope and a ground-based visible band telescope are comparable, ${ }^{3}$ we chose to build a visible demonstration system despite the stringent precision requirements for a visible interferometer. Progress during the course this program has been reported previously., 4 An earlier infrared imaging Fourier transform spectrometer was described by Maillard et $a{ }^{6}{ }^{6}$ and a mid-infrared IFTS, which influenced our work has been described by Bennett et al. ${ }^{7}$

Since the spectral resolution is flexible, observations can be tailored to the type of object under study. For example we have performed low resolution observations to determine the total optical emission of a galaxy cluster and the photometric redshift of individual cluster members. The advantage of the IFTS is that no preselection of objects is required for fabrication of a slit mask or optical fiber placement. As such, more individual galaxies within the cluster may be observed leading to better statistics for formulation of the massto-light ratio. Preliminary high resolution observations have also been conducted to determine the radial velocity profiles of individual galaxies where an advantage of the IFTS is that the errors due to the failure to exactly place the slit at the galaxy center are eliminated. Other classes of measurements for which the IFTS may prove valuable are observations of gravitational weak lensing, the chemical abundance distribution of nebulae and the classification of stars in globular clusters.

wishnow@llnl.gov, LLNL L-043, 7000 East Av., Livermore CA 94551, USA 


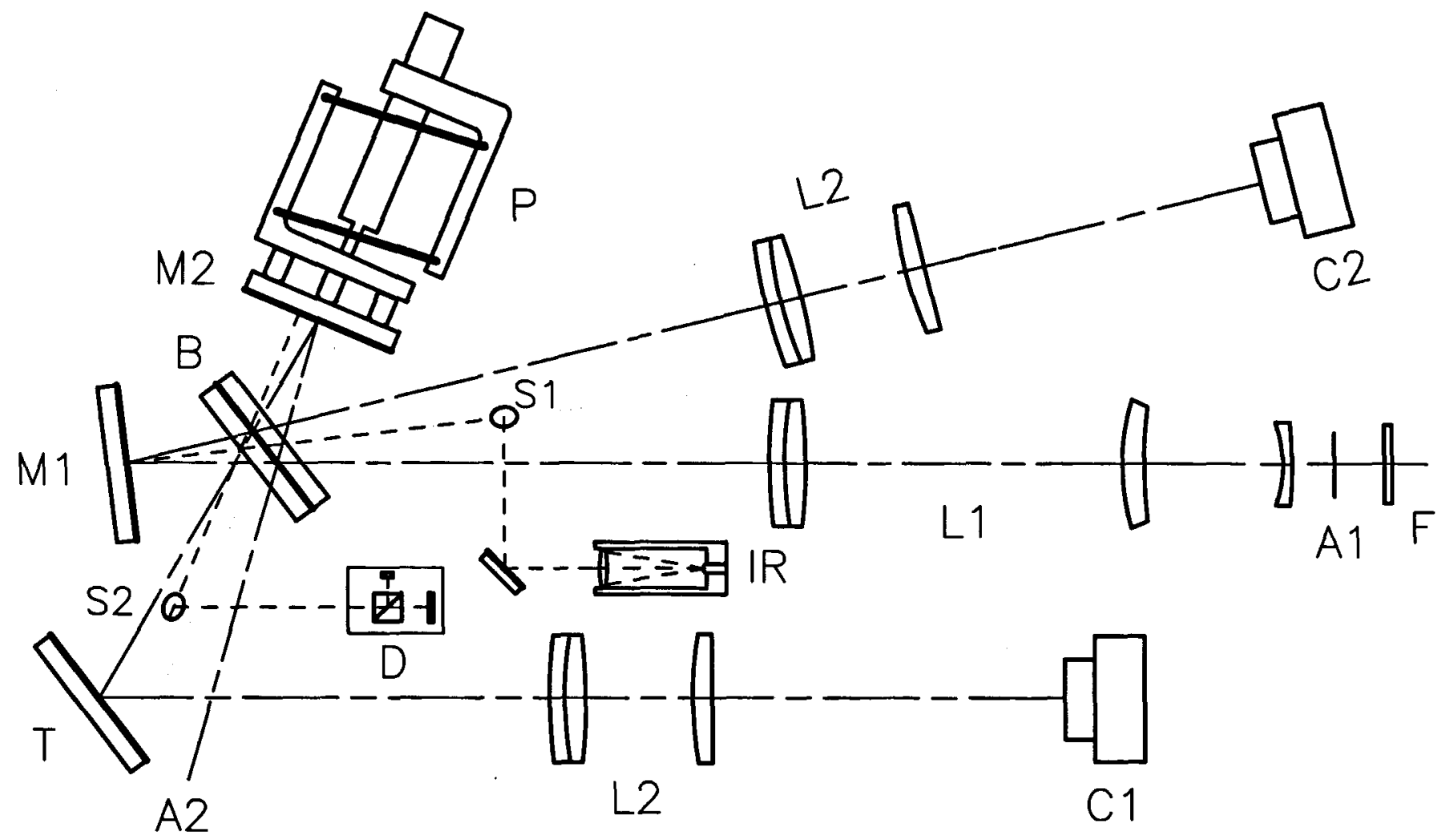

Figure 1. Schematic diagram of the instrument. Radiation from the telescope passes through a filter $F$ and is focussed at A1. The light is collimated, arrives at the beamsplitter B where one component traverses the path to M1, while the other takes the path to M2 (the moving mirror). The beams recombine at B and images are recorded by the cameras $\mathrm{C} 1$ and C2. The IR metrology system is in the center of the interferometer. See text for details.

\section{THE INSTRUMENT}

\subsection{Overview}

The instrument is a dual-input/dual-output differential Michelson interferometer. Our instrument differs from typical Fourier transform spectrometers in two important ways: the detectors are CCD cameras rather than single element detectors, and the optical path is scanned in discrete steps rather than scanned continuously. Two CCD cameras record images over a series of uniform optical path length differences. The camera images are combined to form a "visibility" interferogram for each pixel. The Fourier transforms of each pixel provide a data-cube with two dimensions of spatial information and one dimension of spectral information.

A diagram of the instrument is shown in Fig. 1. Radiation from the telescope passes through the bandpass defining filter $\mathrm{F}$ and arrives at the focus $\mathrm{A}$. The light is then collimated by the lens system $\mathrm{L} 1$. The collimated beam arrives at 7 degrees off-axis to the interferometer, a Michelson interferometer with a beamsplitter B which is 30 degrees from normal. The visible beamsplitter surface is between the two glass plates and it has approximately $50 \%$ reflection from 420 to $700 \mathrm{~nm}$. M1 is a fixed mirror and M2 is a moving mirror which is translated by the porch-swing, $P$. The beam that is initially reflected by the beamsplitter is reflected by M2 and is then transmitted by the beamsplitter, interferes with the beam which is transmitted, then reflected. The combined beam then passes to the turning mirror, T, and is focussed by the camera lens system L2 onto C1, camera 1. The beam which is transmitted-transmitted by the beamsplitter interferes with the beam which is reflected-reflected; this light leaves the interferometer through the entrance port, is focussed by camera lens L2, and is recorded by the second camera C2. Each camera records an interferogram which is proportional to 
the difference between the spectrum of the telescope input A1 and the second input A2 which is blocked. The cameras $\mathrm{C} 1$ and $\mathrm{C} 2$ record fringes which are out of phase with respect to each other.

The moving mirror is servo-controlled by monitoring the interference pattern of an infrared laser, IR, which is introduced to the interferometer, on-axis, via a small spider mounted mirror, S1. The laser light is split by the beamsplitter surface at the far left. The interfering laser beams are reflected by the spider mirror, S2, to a set of IR diode arrays, D. A computer system monitors these diode arrays to adjust both the optical path difference and tip-tilt piezo-electric transducers on the porch-swing.

\subsection{Optical system}

The collimating and camera lenses have identical focal lengths and form an optical system with unity magnification. The diameter of the collimated beam from a point source is $52 \mathrm{~mm}$. The optical system was designed to match the $\mathrm{f} / 10$ Nasmyth focus of the $3.5 \mathrm{~m}$ telescope at the Apache Point Observatory. The cameras are made by Pixelvision and have high quantum efficiency SITe CCDs with $1024 \times 1024,24$ micron pixels. The cameras are binned $2 \times 2$ yielding a plate scale of 0.28 arcseconds per pixel. A seeing-limited point spread function of 0.7 arcseconds FWHM therefore extends linearly over 2.5 pixels. The camera fields of view are 2.4 arcminutes on a side. The lens systems have minimal chromatic aberration over the wavelength range of the beamsplitter coating. The details of the lens systems will be discussed in a future paper.

The beamsplitter is composed of two BK7 plates, flat to within $1 / 10 \lambda$ at $633 \mathrm{~nm}$, that are $107 \mathrm{~mm}$ in diameter and $12.5 \mathrm{~mm}$ thick; they are cemented together using Norland 61 optical epoxy. The visible beamsplitter coating is between the two plates, the IR beamsplitter coating has low reflectivity in the visible and is located on the outside surface of one of the plates.

The components of the interferometer, including the infrared metrology system, are mounted on and within a large solid piece of aluminum. The interferometer lenses and cameras are mounted on a hex-core aluminum plate which is reinforced by 12 inch high aluminum "U-channel" beams. The optical system is stable to within a fraction of a pixel as the system rotates on the Nasmyth bearing of the telescope. The image shift over the course of an observation due to rotation is not observable as errors in tracking are larger.

\subsection{Interferometer}

The moving mirror is borne on a precision porch-swing mechanism. Coarse movement is provided by a Burleigh inchworm system and precision adjustment of the optical alignment and path difference is accomplished by piezo-electric actuators. The control system is based on the adjustment of the moving mirror in accordance with the fringe pattern generated by a distributed feedback laser operating at a wavelength of $1.549 \mu \mathrm{m}$ and recorded by linear diode arrays. The mirror servo control is performed by a dedicated microcomputer with a loop frequency of about $100 \mathrm{~Hz}$. The computer system must not only hold a given position during integration but it must move to the next optical path position in a controlled manner. The interferometer was designed and built by ABB Bomem; we have significantly modified the physical components and the servo-control algorithms in order to perform astronomical observations.

Precise servo control is critical to the operation of a Fourier transform instrument. By placing the metrology beam through the center of the interferometer we insure that there is a known correspondence between the optical path determined by the metrology beam and the science beam. As a consequence, it is extremely important that the cameras do not record light from the laser as it is vastly brighter than star light, and for this reason we use a laser with a wavelength at which the cameras are nearly insensitive. We additionally block light from the laser which scatters from the edges of lens to the cameras. Because we are basing interferogram sampling on fractions of an infrared laser wavelength, the laser frequency must be very stable.

It is well known that the environment at the telescope is harsh. This is particularly true for an interferometer which must maintain alignment to a fraction of an optical wavelength. Some of the difficulties associated with building an astronomical interferometer are: the instrument orientation varies with respect to gravity during observations, the temperature can be very cold, wind buffeting and motor rumble can vibrate sensitive components, and electrical noise from motors can contaminate servo-control signals. We have encountered and addressed these issues. The interferometer moving mechanisms are very stiff so that the resonant frequency is 


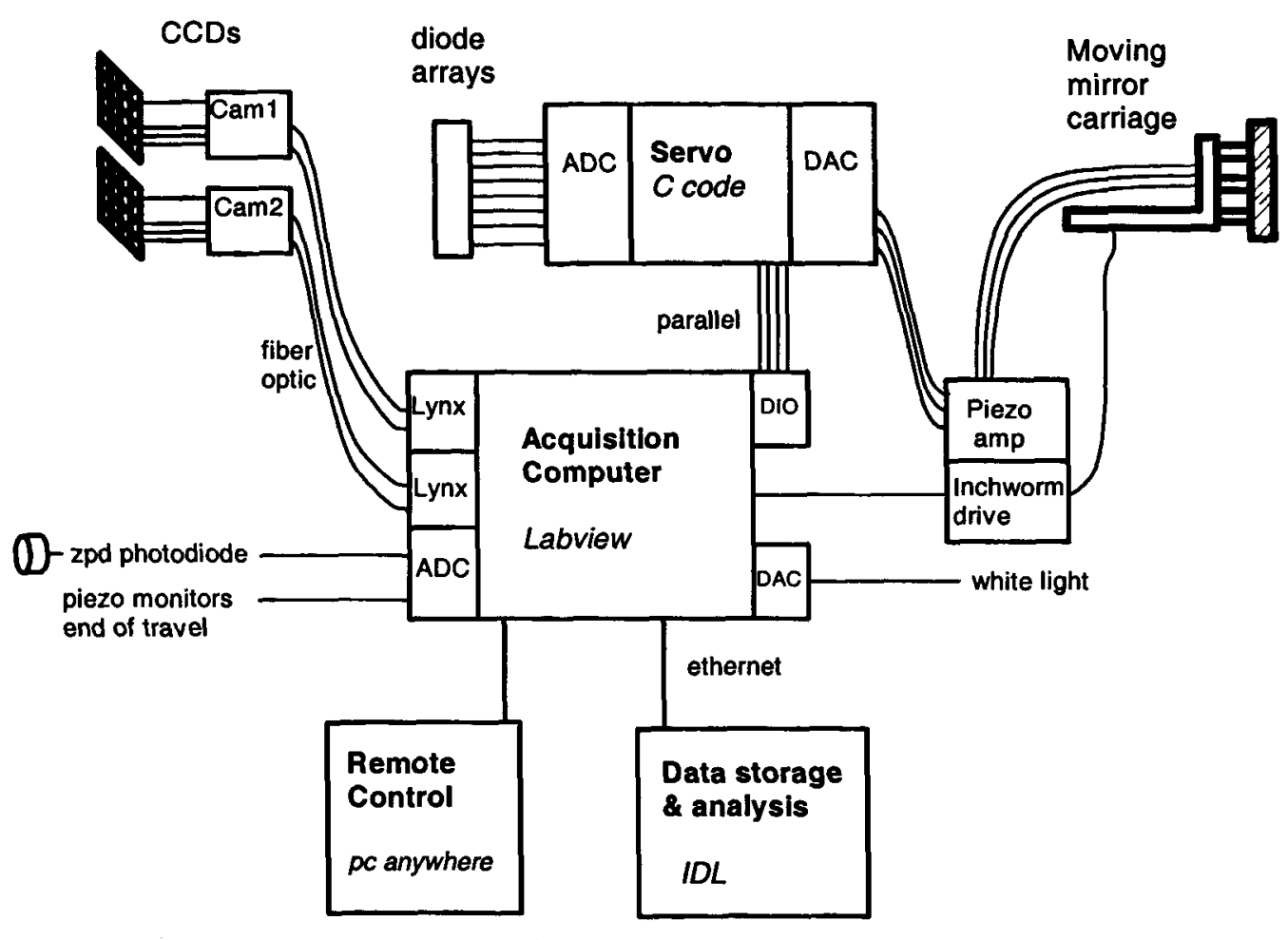

Figure 2. Diagram of the computer control and acquisition system.

above motor rumble. The interior of the optical bench is thermally insulated, and the interferometer section is further isolated to obtain a uniform temperature. To avoid thermally generated turbulence, there are no heat sources within the interferometer enclosure.

\subsection{Computer system}

The control of the interferometer requires a system of computers which is shown in Fig. 2. The interferometer alignment servo system is controlled by a dedicated single board computer with a 32 channel 12 bit ADC and 8 channel DAC. Signals from the diode arrays are received at the ADC and calculated output signals are sent to the piezo amplifiers and then to the moving mirror piezo transducers. The servo computer is mounted on the optical bench support structure. Digitized images from the cameras are sent via fiber optics to two Pixelvision "Lynx" framegrabber cards connected to the acquisition computer. This computer also controls the large scale movement of the moving mirror and ancillary systems such as mirror end-of-travel switches, a white light source and receiver, and instrument monitoring. The acquisition computer sits on the observatory dome floor. Finally the acquisition computer writes FITS image files via ethernet onto the disk of the storage and analysis computer in the control room. Another computer running the "pcAnywhere" program can be used to remotely access the acquisition computer from the telescope control room.

\section{OBSERVING WITH THE INTERFEROMETER}

\subsection{Observing parameters}

The nature of the astronomical observation determines the camera exposure times and the parameters of the interferometric scan. The desired resolution $\mathrm{R}$, or alternatively the number of spectral channels across the band, gives the ultimate scan length $\mathrm{L}=\mathrm{R} / \sigma$ where $\sigma$ is the central frequency in wavenumber units $(\sigma=1 / \lambda$ in $\mathrm{cm})$ of the spectral region to be studied. The physical motion of the moving mirror, $\pm 5 \mathrm{~mm}$, leads to a maximum resolution at $18182 \mathrm{~cm}^{-1}(550 \mathrm{~nm})$ of $\sim 18000$, or $17 \mathrm{~km} / \mathrm{sec}$. 
In general, the Nyquist sampling criterion determines the distance between samples $h=1 /\left(2 \sigma_{\max }\right)$ where $\sigma_{\max }$ is the maximum optical frequency of interest. Because the product of the number of exposures and the time for each exposure is limited, as with all astronomical observations, the interferometer is often used with sampling intervals which are larger than Nyquist sampling. Valid data is still obtained because the band is limited by optical filters and because the interferometer does not scan continuously. This procedure is analogous to working in higher orders of a grating spectrometer and will be referred to as higher order sampling. The trade-off for working in higher orders is that the optical bandwidth must be narrowed so that the entire band falls within one order of the interferometer. We have developed observer software tools to easily determine the desired parameters. Table 1 gives some examples of sampling parameters that have been used. Odd numbers of samples are data sets that were truncated due to problems, generally the weather.

Table 1. Parameters used for various types of observations. Sampling interval is in fractions of the IR laser wavelength. For narrow band filters, the bandpass is given as width and (band-center).

\begin{tabular}{|c|c|c|c|c|c|}
\hline samp. interval. & no. of samps. & bandpass & resolution & Res. at $\mathrm{H} \alpha$ & int. order \\
\hline $1 / 16$ & 256 & $420-680 \mathrm{~nm}$ & $406 \mathrm{~cm}^{-1}$ & 175 & 1 \\
$1 / 8$ & 128 & $420-680$ & 406 & 175 & 1 \\
$1 / 4$ & 64 & $420-680$ & 406 & 175 & 2 \\
$1 / 8$ & 1024 & $420-680$ & 51 & 300 & 1 \\
$1 / 4$ & 512 & $420-680$ & 51 & 300 & 2 \\
2 & 479 & $2.5(656.3)$ & 6.8 & 2241 & 10 \\
10 & 69 & $2.5(656.3)$ & 9.4 & 1614 & 47 \\
21 & 70 & $1.5(661.5)$ & 4.4 & 3446 & 98 \\
\hline
\end{tabular}

Each ray of the science beam passes through the interferometer at an angle $\theta$ with respect to the metrology laser, and the optical path difference for that ray is reduced from the metrology path by a factor of $\cos \theta$. For low resolution scans only the 7 degree angle difference between the metrology beam and science beam is relevant, but for high resolution scans the optical path difference due to different angles to pixels on either side of the CCD must be taken into account. The ultimate resolution of an IFTS is limited by the requirement that interference rings be larger than 2 pixels, ${ }^{2}$ but this interferometer does not operate near this limit.

\subsection{Operations}

The interferometer is aligned by eye prior to each data set by injecting a divergent visible laser at the location of the telescope focus and tuning the piezo tip and tilt actuators to obtain a uniform fringe across the beamsplitter. A white light source is turned on and the moving mirror is positioned at the zero path difference (z.p.d) location. The laser and white light are turned off and the optical path is scanned by the computer over a distance of two IR laser optical fringes and a table of fringe values is obtained which establishes the error signal for the servo system. The user now sets the exposure time, the number of exposures and the distance between successive samples, and the acquisition computer backs up the moving mirror to $1 / 2$ of the scan distance and begins the series of exposures. At the end of a data set the computer returns the moving mirror to the zero path difference position.

Two problems arose when working at low temperatures. The beamsplitter distorts at temperatures below about $12 \mathrm{C}$; this is either due to the mounting scheme or the optical epoxy. In addition, the Inchworm mechanism does not clamp properly at temperatures near $5 \mathrm{C}$ and below. To alleviate these problems, the interferometer enclosure is heated using an electric blanket powered by a DC supply. Since the heat is applied externally, no turbulence is introduced in the interferometer. The beamsplitter assembly is being redesigned with a new mount and optical coupling fluid rather than epoxy. 


\section{PERFORMANCE}

\subsection{Throughput}

The transmission of the interferometer was measured in the laboratory. An $\mathrm{f} / 10$ laser beam was injected at the interferometer input and a ratio of the power at the input, to the sum of the power received at each camera position, was measured with a calibrated photodiode. The measured transmission was $47 \%$ for both a red laser and green laser. The overall efficiency of the interferometer system, including the general blocking filter (420-680 $\mathrm{nm}$ ) of $73 \%$ transmission and the average CCD quantum efficiency (Q.E.) over this band of $80 \%$, is therefore $27 \%$.

The transmission of the interferometer was confirmed by observing the standard star GD71 using the APO 3.5 meter telescope and the facility camera SPICAM through the MSSSO R filter. The same star was immediately observed using the interferometer mounted on the same telescope through a Kron-Cousins $\mathrm{R}$ filter. The stellar flux measured by the IFTS was $56 \%$ that of SPICAM. Given that product of the CCD Q.E. and filter transmission for SPICAM is approximately 1.12 times that of the IFTS, the transmission of the IFTS is $63 \%$ that of SPICAM. If SPICAM is estimated to have an optical transmission of $80 \%$ (including the filter), the transmission of the IFTS through the same filter is $50 \%$, similar to the measurement of $47 \%$ above.

\subsection{Low resolution spectroscopy}

The interferometer frequency axis is calibrated by placing an integrating sphere fed by an optical fiber from an attenuated HeNe laser at $632.8 \mathrm{~nm}$ wavelength at the input of the interferometer. The interferometer is scanned over 256 steps where each optical path delay is $1 / 16$ of the IR laser wavelength times the cosine of 7 degrees. The left panel of Fig. 3 shows a segment of the interferograms the from the two cameras and the visibility from a 10 pixel radius region as a function of frame number. The visibility is

$$
v=\frac{(a / m a-b / m b)}{(a / m a+b / m b)} \times(m a+m b)+(m a+m b)
$$

where $a$ and $b$ are the camera 1 and 2 signals, and $m a$ and $m b$ are the median values of $a$ and $b$. The visibility function balances the gain differences between the two cameras and the integrated visibility function is equal to the sum of the integrated signals of camera 1 and camera 2; it therefore preserves the total number of photons received by the interferometer. The data shown in Fig. 3 was recorded in the observatory dome, but the instrument was not mounted on the telescope. Only the central portion of the 256 point interferogram is shown.

The right panel of Fig. 3 shows the modulus of the Fourier transform (the power spectrum) of the full 256 frame visibility interferogram. The peak frequency lies between the frequencies 15448 and $15854 \mathrm{~cm}^{-1}$, or between 647.3 and $630.7 \mathrm{~nm}$ as is expected. The spectral value at zero frequency contains the average value of the interferogram. The small side peaks in the spectrum are due to errors in sampling that are analogous to "ghosts" observed in spectra from grating instruments. These sampling errors are quite small in the laboratory, but they are greater when at the telescope and the instrument is subject to vibration. We have continued efforts to reduce these "sidelobes" and work presented here was obtained prior to alleviating some vibration induced problems.

\subsection{Stellar standards}

The main astronomical program for the IFTS involves relatively low spectral resolution observations of galaxy clusters. We have observed standard stars at comparable resolution and the observations of G191-B2B are presented in Fig. 4. The left panel shows the two camera signals and the visibility function derived from the total number of electrons received over a radius of 20 pixels centered on the star where the average sky background has been subtracted.

The right panel of Fig. 4 shows the power spectrum and the phase from the transform of the visibility. The optical band is clearly seen between 14500 and $24000 \mathrm{~cm}^{-1}$ or 690 to $417 \mathrm{~nm}$, where the shape of the band is determined by the band defining filter. Small anomalous "sidelobes" are observed above and below the optical 

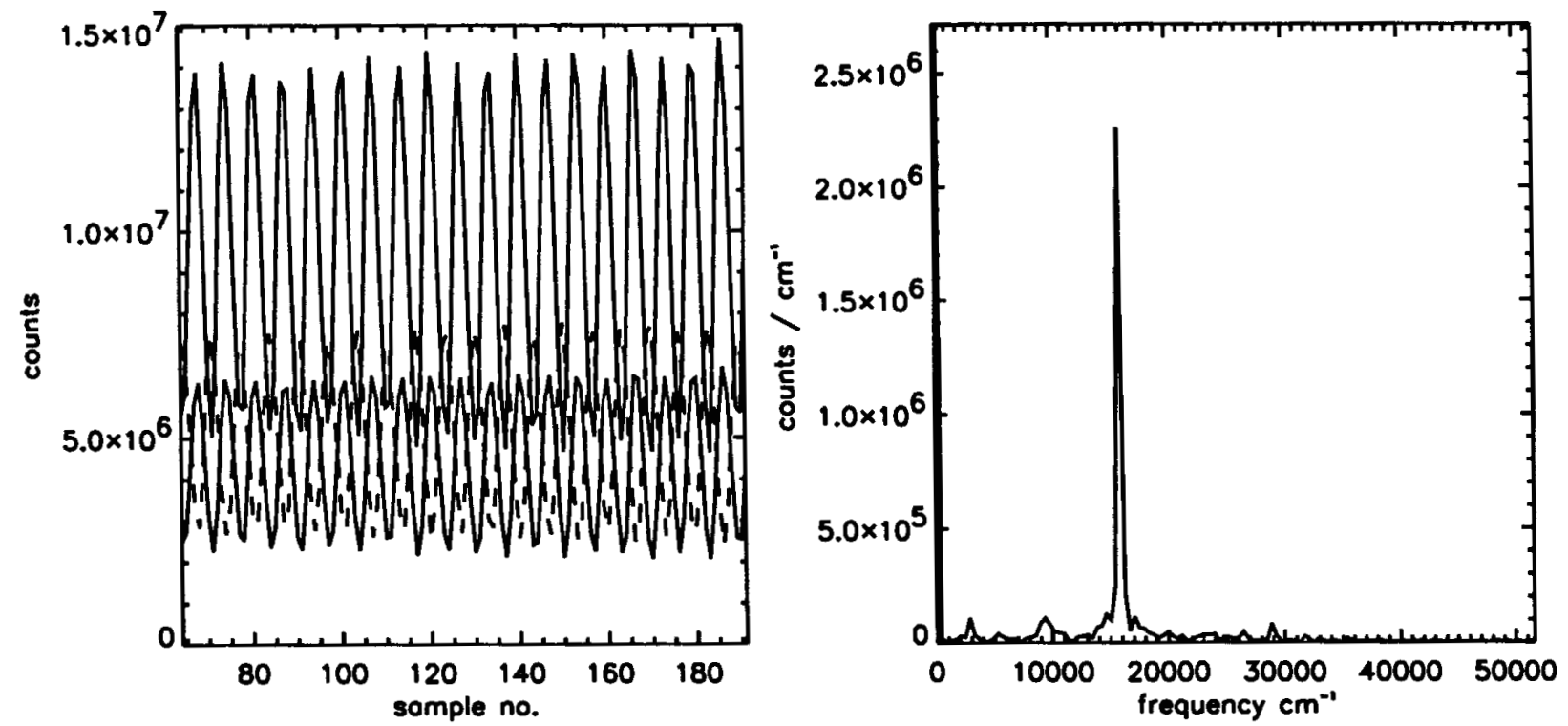

Figure 3. Left panel: the central portion of 256 point interferograms for camera 1 (lower solid), camera 2 (dashed) and the visibility (upper solid). Each camera signal is the sum of a 10 pixel radius for a HeNe laser filling an integrating sphere at the entrance aperture. Right panel: the power spectrum (upper) that results from the Fourier transform of the visibility. The small peaks in the wings of the main spectral line are artifacts due to sampling errors.
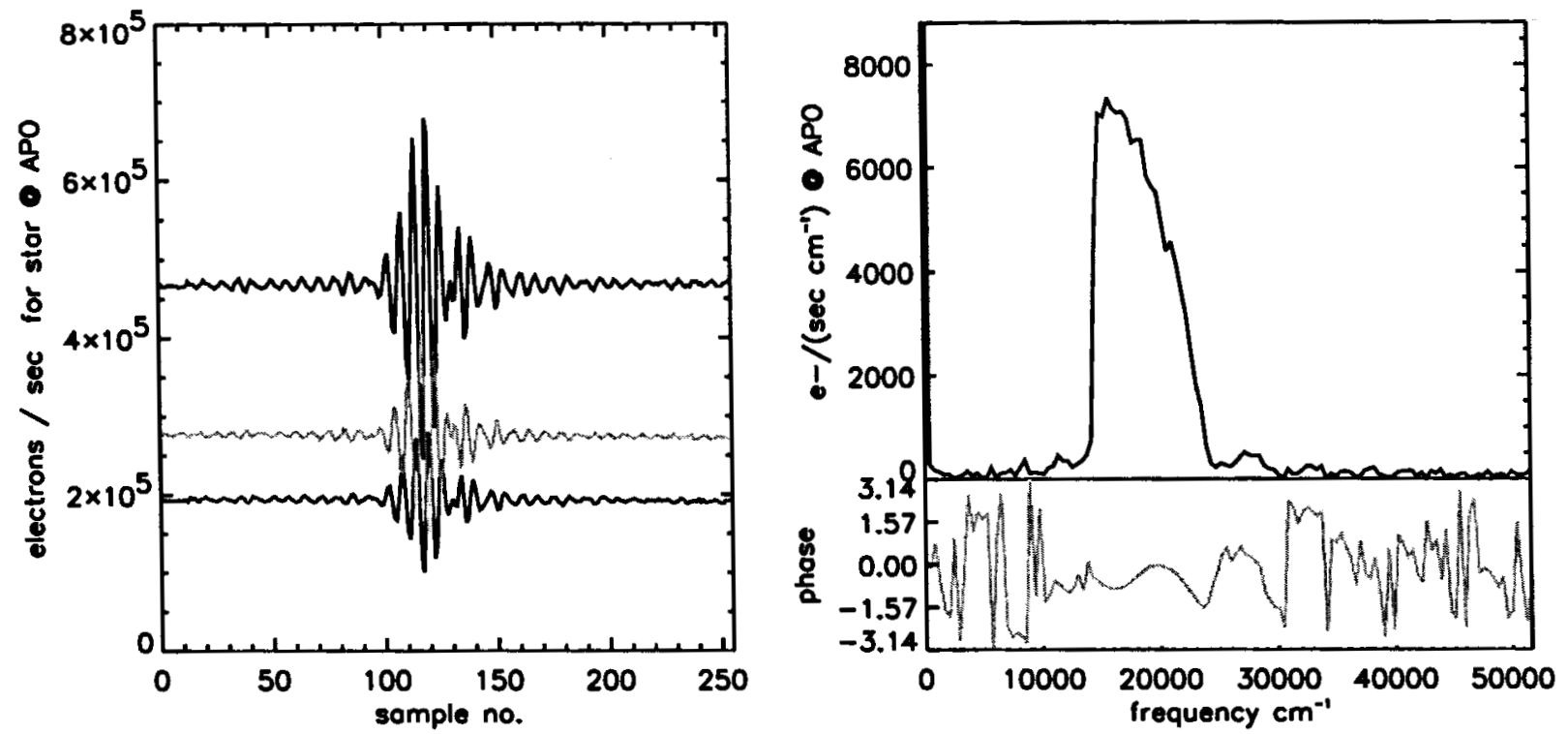

Figure 4. Left panel: camera 1 (solid), camera 2 (grey) and visibility (upper solid) for a 20 pixel radius for the star G191-B2B. Right panel: the upper plot shows the power spectrum and the lower plot shows the phase spectrum for the visibility function. The optical band is between 14500 and $24000 \mathrm{~cm}^{-1}$ or $690-417 \mathrm{~nm}$. 

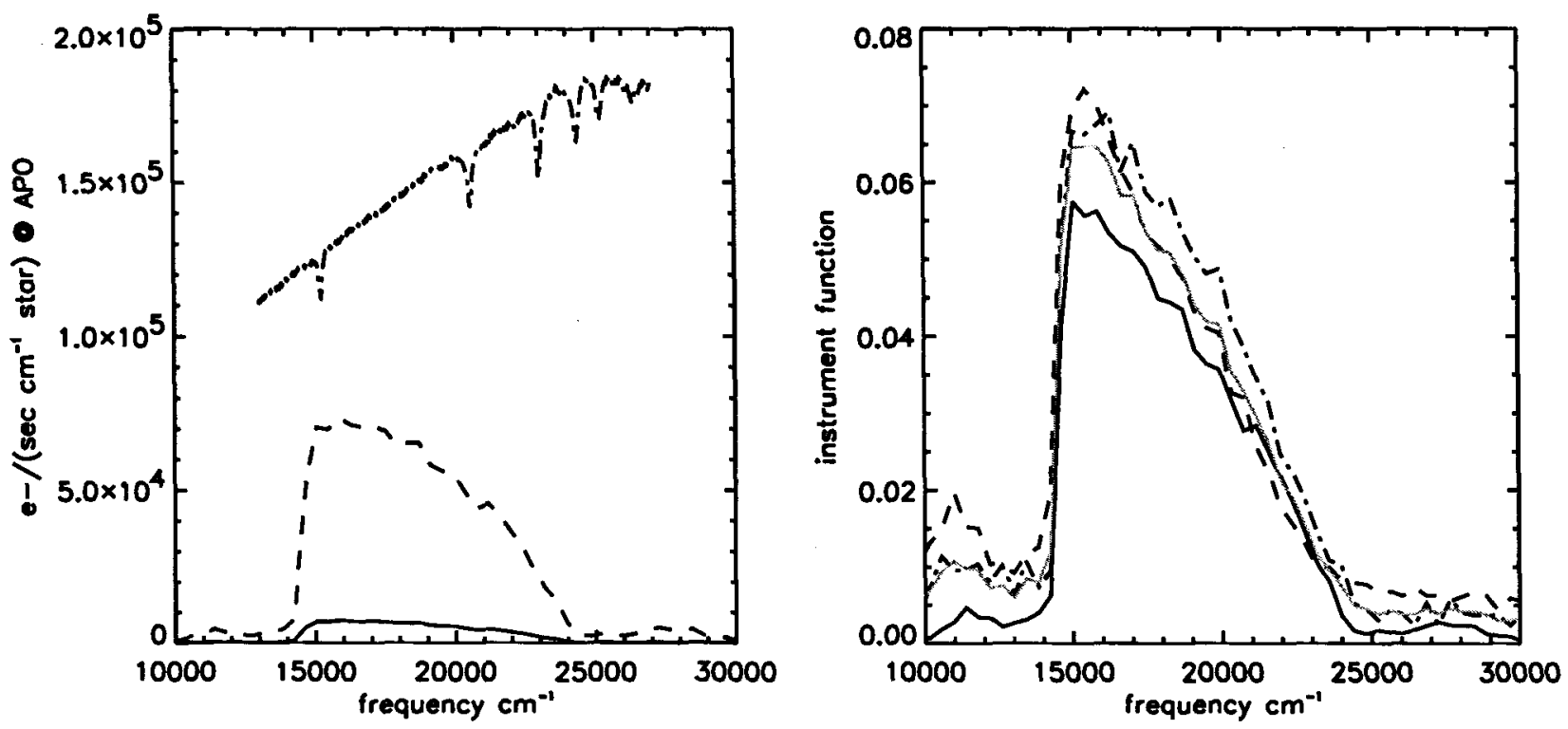

Figure 5. Left panel: the measured spectrum (solid), measured spectrum x10 (dashed) and the Oke measurement in wavenumber units (dot-dashed) for the star G191-B2B. Right panel: Instrument transmission functions derived from 3 different measurements of G191-B2B and the average instrument transmission function (grey line).

band. The phase plot is noisy over frequency regions with no low optical power. Over the optical bandpass the phase is a smooth function that results from the optical properties of the beamsplitter. These phase shifts as a function of frequency give the asymmetric shape of the visibility interferogram in the left panel.

The left panel of Fig 5 compares the stellar spectrum, and the spectrum multiplied by 10, to the published spectrum of G191-B2B. ${ }^{8}$ The published spectrum is corrected for atmospheric extinction, but the measurements are not; the measurements were obtained when the star was at an airmass of $\sim 1.56$. The standard spectrum is converted to wavenumber units and electrons per second per frequency interval for the APO 3.5 meter aperture telescope assuming a Q.E. of 1 . The IFTS instrument transmission function is obtained by dividing the measured spectrum by a smoothed version of the standard spectrum. The right panel of Fig. 5 shows three instrument transmission functions derived from different measurements of the star. The amplitude differences of the functions reflect the differences in the modulation of the visibility. These differences are due to the quality of alignment or degree of instrument vibration during observations. The degree to which "sidelobes" appear also varies from measurement to measurement.

The average instrument transmission function has a peak value of approximately $6.5 \%$. The actual efficiency is a factor of 2 greater than this, as signal recorded, but appearing at "negative" spectral frequencies, has not been included in determining the instrument transmission. The further difference from the $27 \%$ efficiency determined from the instrument optical transmission mentioned above is due to an average interferogram modulation of approximately $50 \%$.

Fig. 6 shows two more stellar standards measured at low spectral resolution. In both cases the interferograms were not of the same length as those above and the visibility function was extended to 256 points using the average value of the visibility function (generally called "zerofilling"). The left panel compares a measurement of the star BD+75,325 with a smoothed published spectrum multiplied by the instrument transmission function derived above. The right panel compares a measurement of the star Feige 34, obtained four months after the observation of G191-B2B, to the Oke spectrum multiplied by the instrument transmission. Over the central region of the band the correspondence between the measurements and the standard spectrum is fairly good, but differences at the band edges are due to changes in the modulation and possibly changes in the filter temperature. Errors in the edge shape can also result from the zerofilling interpolation process. The correspondence of the 

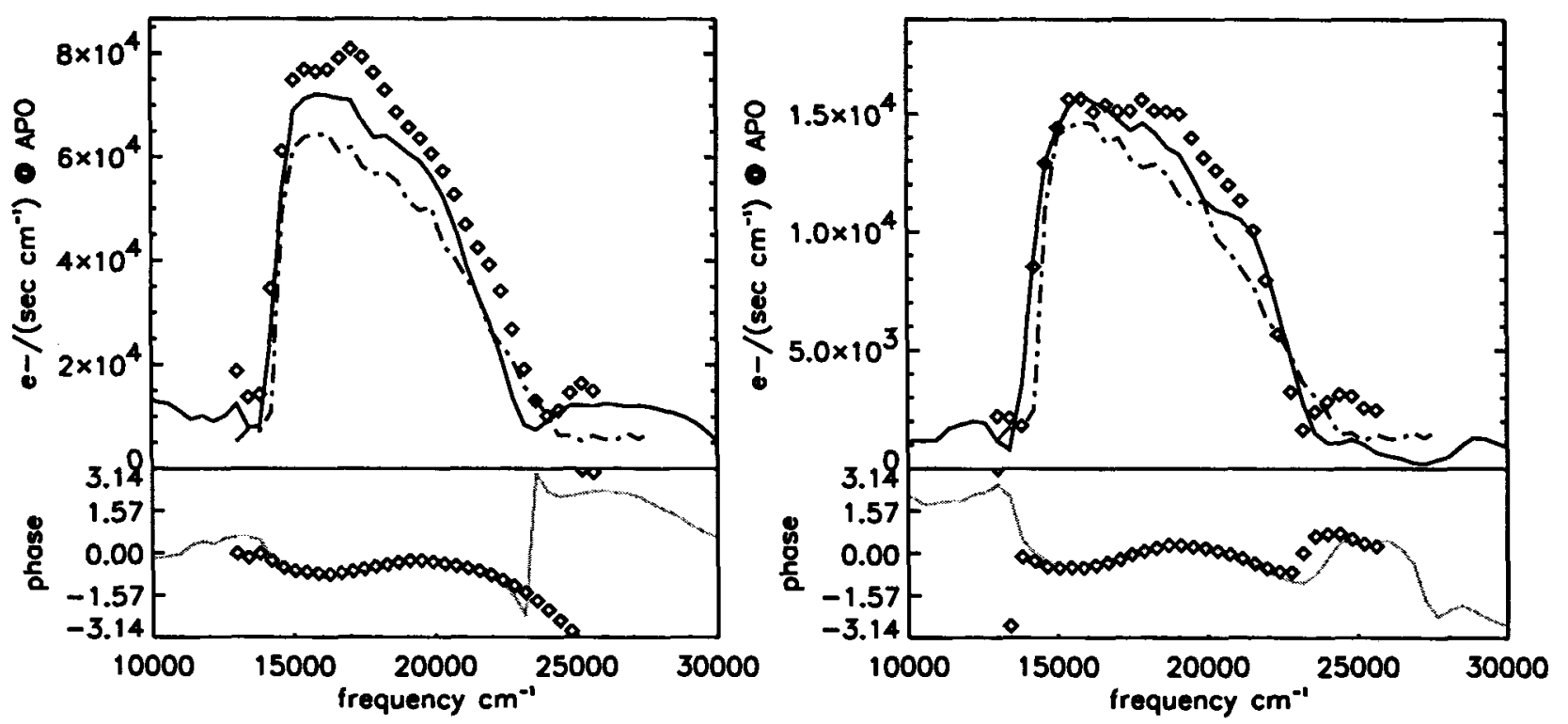

Figure 6. Left panel: the measured spectrum in 1st (solid) and 2nd order (diamonds) and the Oke measurement in wavenumber units multiplied by the instrument transmission function, for the star $\mathrm{BD}+75,325$; the lower portion shows the 1st and 2nd order phase spectra. Right panel: the measured spectrum in 1st (solid) and 2nd order (diamonds) and the Oke measurement in wavenumber units multiplied by the instrument transmission function (dot-dashed) for the star Feige34; lower portion is 1st and 2nd order phase spectra.

data in the right panel indicates that the instrument is relatively stable over long periods of time and two trips between LLNL and APO.

The diamonds denote spectra which are obtained from the same data using every fourth point, or using the interferometer in second order. The differences in the spectra between the diamonds and the solid line show the errors which occur when sampling below the Nyquist frequency. The differences arise from the inclusion of false sidelobe intensity into the band; this is an example of spectral "aliasing." The lower plots show the phase spectra and a comparison to the phases derived from the 2nd order transforms. The plots demonstrate that data obtained using the interferometer in 2 nd order is generally valid. In order to reduce the variation in the instrument transmission function, we are continuing efforts to reduce the interferometer susceptibility to vibration. We expect that data obtained with consistent modulation efficiency and little power in the sidelobes should match stellar standards even better than what is displayed here.

\subsection{Datacube of the Orion nebula}

An illustration of the capabilities of the IFTS is given by a data set of the Orion nebula. The image is 2.4 arcminutes on a side. The interferogram consists of 1024 frames each with a 1 sec exposure. The optical path difference between each frame was $1 / 8$ of the IR laser wavelength $(x \cos \theta$ of the angle through the interferometer associated with a particular point in the image). The picture on the left is the panchromatic image. The image is $512 \times 512$ pixels and each pixel has 512 spectral elements; this gives a total of 60 million spectral elements in the visible region of the datacube. The right panel shows spectra extracted from four locations in the datacube. Each spectral plot is an average over a $3 \times 3$ pixel region. The prominent peaks are due to the $\mathrm{H} \alpha$, OIII and $\mathrm{H} \beta$ emission lines. The $\mathrm{H} \alpha$ line has nearly the same flux at locations A, B and C, but the OIII lines are stronger at $\mathrm{A}$ than the other points. The largest $\mathrm{H} \alpha / \mathrm{OIII}$ ratio is found at point $\mathrm{C}$. The picture and plots show the potential of full field imaging and spectroscopy that is realized with the IFTS. 

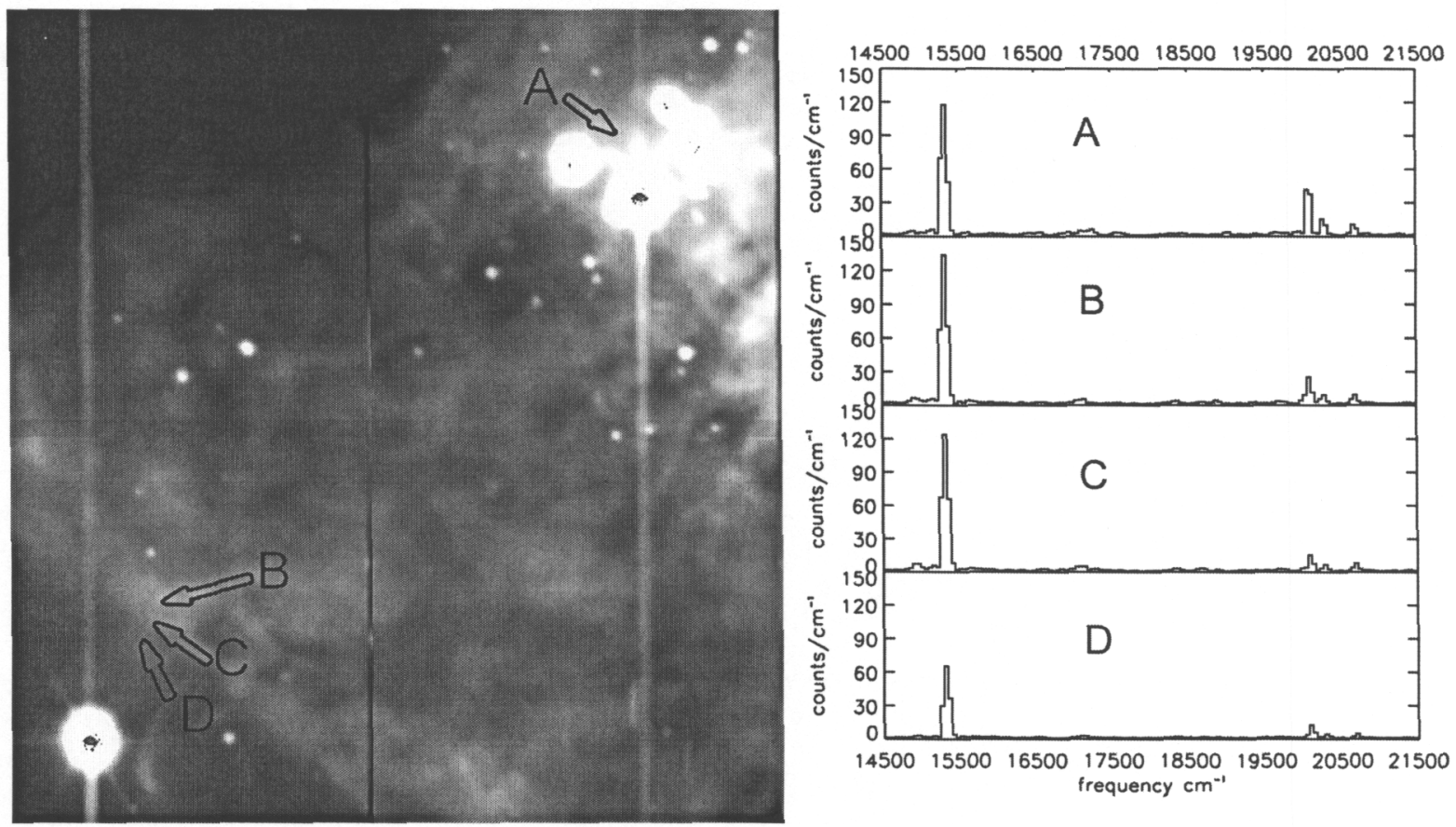

Figure 7. Left panel: the panchromatic image of the orion nebula. Letters denote locations of spectra. Right panel: the spectra from four locations. The lines observed are $\mathrm{H} \alpha\left(15237 \mathrm{~cm}^{-1}\right)$, OIII (19972 and $\left.20165 \mathrm{~cm}^{-1}\right)$ and $\mathrm{H} \beta(20572$ $\left.\mathrm{cm}^{-1}\right)$.

\section{ACKNOWLEDGMENTS}

We would like to thank Russ Alexander of the Canadian Space Agency and Jacques Giroux of Bomem for sponsoring the development of the interferometer, and James Graham of UC Berkeley, Charles Bennett of LLNL, Mark Abrams of ITT and Andre Villemaire of Bomem for their involvement in the initial stages of this project. This work was based on observations made with the Apache Point Observatory 3.5-meter telescope, which is owned and operated by the Astrophysical Research Consortium. We would like to thank the staff of APO, in particular, Russett McMillan, Craig Loomis and Mark Klaene. This work was performed under the auspices of the U.S. Department of Energy, National Nuclear Security Administration by the University of California, Lawrence Livermore National Laboratory under contract no. W-7405-Eng-48.

\section{REFERENCES}

1. R. Wurtz, E.H. Wishnow, S. Blais-Ouellette, K.H. Cook, B. Holden, D. Carr, C.W. Stubbs, "Gallery of datacubes obtained with the Livermore imaging Fourier transform spectrometer, " in Specialized Optical Developments in Astronomy, E. Atad-Ettedgui, ed., Proc. SPIE, 4842, 2002.

2. J. Graham, M. Abrams, C. Bennett, J. Carr, K. Cook, A. Dey, J. Najita, E. Wishnow, "The Performance and Scientific Rationale for an Infrared Imaging Fourier Transform Spectrograph on a Large Space Telescope", $P A S P, 110,1205-1215,1998$.

3. H.S. Stockman, The Next Generation Space Telescope: Visiting a Time When Galaxies Were Young, AURA, Washington D.C., 1997.

4. R. Wurtz, K.H. Cook, C.L. Bennett, J. Bixler, D. Carr, E.H. Wishnow, "Ground Based Demonstration of Imaging Fourier Transform Spectrometry and Techniques," ASP conf. ser., 207, E.P. Smith and K.S. Long eds., 203, 2000. 
5. R. Wurtz, E. Wishnow, S. Blais-Ouellette, K. Cook, D. Carr, I. Lewis, F. Grandmont, C. Stubbs, "Astronomy with a Visible-Band Imaging Fourier Transform Spectrometer," Next Generation Wide Field Multiobject Spectroscopy, ASP conf. ser., in press, 2002.

6. J.P. Maillard, "3-D Spectroscopy with a Fourier Tranform Spectrometer," Tridimensional Optical Spectroscopic Methods in Astrophysics, G. Comte, M. Marcelin, eds., ASP conf. ser., 71, 316, 1995.

7. C.L. Bennett, M. Carter, D.J. Fields, J.A. Hernandez, "Imaging Fourier Transform Spectrometer,", Imaging Spectrometry of the Terrestrial Environment, G. Vane, ed., Proc. SPIE, 1937, 191, 1993.

8. J.B. Oke, "Faint spectrophotometric standard stars," $A J$, 99, 1621, 1990. 